\title{
COVID-19 Associated Hemophagocytic Lymphohistiocytosis and Coagulopathy: Targeting the Duumvirate
}

\author{
Sukrita BhattacharJee ${ }^{1}$, Mainak BanerJeE ${ }^{2}$ and Rimesh PaL ${ }^{3}$ \\ From ${ }^{1}$ Institute of Hematology and Transfusion Medicine, Medical College and Hospital, and ${ }^{2}$ Institute of Post Graduate Medical \\ Education and Research, Kolkata, West Bengal, India; and ${ }^{3}$ Post Graduate Institute of Medical Education and Research, \\ Chandigarh, India. \\ Correspondence to: Dr Sukrita Bhattacharjee, Department of Hematology, Institute of Hematology and Transfusion Medicine, \\ Medical College and Hospital, Kolkata,India.skrtbhttchry@gmail.com
}

\begin{abstract}
Context: Preliminary data on coexistence of secondary hemophagocytic lymphohistiocytosis syndrome $(\mathrm{HLH})$ and disseminated intravascular coagulation (DIC) in critically ill children with novel coronavirus disease (COVID-19) are emerging. Herein, we summarize the available literature and fill-in the gaps in this regard.

Evidence Acquisition: We have performed a literature search for articles in PubMed, EMBASE and Google Scholar databases till May 12, 2020, with following keywords: "COVID-19", "SARS-CoV2", "HLH", "HScore", "coagulopathy", "D-dimer", "cytokine storm", "children" and "pediatrics" with interposition of Boolean operator "AND".

Results: Children presenting with moderate-severe COVID-19 and Kawasaki disease shock-like syndrome exhibit peripheral blood picture analogous to HLH. HScore, a validated tool to diagnose HLH, has been suggested to screen severe COVID-19 patients for cytokine storm. However, HScore faces certain
\end{abstract}

$\mathrm{N}$ ovel coronavirus disease 2019 (COVID-19) has emerged as a pandemic, claiming over 350,000 lives worldwide. Disease mortality has been mostly attributed to viral pneumonia, complicated by acute respiratory distress syndrome (ARDS) and/or sepsis. A cytokine storm like picture in peripheral blood, as evident by significantly higher plasma levels of interleukin (IL)-2, IL-7, tumor necrosis factor- $\alpha$ (TNF- $\alpha$ ), granulocyte colonystimulating factor (GCSF), monocyte chemoattractant protein-1 (MCP-1), inducible protein 10 (IP 10) and macrophage inflammatory protein $1-\alpha$ (MIP-1 $\alpha$ ), has been shown in severe COVID-19 illness [1]. Albeit uncommon, but biochemical findings like C-reactive protein, D-dimer, liver enzymes and ferritin in high concentration along with increase in IL-6, IL-10 and interferon (IFN)- $\gamma$ are being reported in critically ill children with COVID-19 [2]. This points towards a coexistence of possible secondary hemophagocytic limitations in this scenario. It may be more pragmatic to use "high D-dimer' ( $>3 \mu \mathrm{g} / \mathrm{mL}$ ) instead of 'low fibrinogen' to facilitate early detection of cytokine storm. COVID-19 associated coagulopathy resembles hypercoagulable form of DIC with bleeding being rarely reported. Although the International Society on Thrombosis and Haemostasis (ISTH) interim guidance recommends low molecular weight heparin in all hospitalized patients, data is lacking in population below 14 years of age. However, in the presence of life-threatening thromboembolic event or symptomatic acro-ischemia, unfractionated heparin (UFH) should be used with caution.

Conclusions: HScore can be used as a complement to clinical decision for initiating immunosuppression. Children with moderate-to-severe COVID-19, especially those with documented thrombocytopenia or chilblains, should be regularly monitored for coagulopathy.

Keywords: Cytokine storm, Disseminated intravascular coagulation, Immunosuppression, Management, SARS-CoV-2.

Published online: June 24, 2020; PII: S097475591600204

lymphohystiocytosis (HLH) syndrome and a form of disseminated intravascular coagulation (DIC). Although biochemical diagnosis of DIC is straightforward, it is often challenging to diagnose HLH in an early evolving stage irrespective of the underlying conditions.

\section{COVID-19-Associated HLH: A Distinct Entity?}

$\mathrm{HLH}$ is a hyperinflammatory state characterized by development of fulminant multi-organ damage (including ARDS), which can also occur secondary to viral infections [3]. Of note, virus-associated secondary HLH (sHLH) was considered in the differential diagnosis of life-threatening pneumonia and SARS-CoV infection during 2003 epidemic [3]. In a recently published series of eight cases [4], authors suggested presence of new entity affecting previously asymptomatic COVID-19 children. A refractory vasoplegic shock like presentation with peripheral edema, pleuro-pericardial effusion, ascites and myocardial involvement was noted. 
Additional presence of variable rash, conjunctivitis, extremity pain along with coronary aneurysm in one child, made authors to consider Kawasaki disease shock syndrome or toxic shock syndrome as differential diagnosis [4]. SARS CoV-2 was only identified in one child post mortem, who died of major ischemic cerebrovascular accident [4]. Although no pathological organism was identified on bronchoalveolar lavage or nasopharyngeal aspirates in seven children; positive antibody tests were later found in ten cases out of more than 20 children presenting in a similar fashion in that center (including eight children of published series). All these eight children, described in the series, had evidence of cytokine storm/HLH like picture in peripheral blood with high D-dimer level (range, 3.4-24.5 $\mu \mathrm{g} / \mathrm{mL}$ ) [4]. Subsequent data regarding this phenomenon are still awaited. Nevertheless, clinicians need to be extremely vigilant to diagnose cytokine storm/HLH in evolving phase in children during this pandemic.

HScore, a widely used tool for diagnosing HLH, was first validated in adult population [5]. Later on, HScore was also found to be more sensitive than adapted HLH2004 guidelines for HLH diagnosis in pediatric population. However, a different cutoff value of the HScore was given for children [6] (Table I). Recently, owing to non-availability of expensive cytokine assays in clinical practice, HScore has been suggested to screen critically ill COVID-19 patients to ensure timely immunosuppression [7]. However, it has certain limitations in this scenario.

Temperature rise, heavily weighted in HScore, does not seem to differ significantly according to the severity of COVID-19 [2,8]. In contrast to most forms of HLH, few studies $[1,8]$ including one study in children [2], reported rather a lower incidence of leukopenia (mostly due to concomitant neutrophilia) in severe cases with cytokine storm. Lymphopenia, the striking hematological abnormality of adults with COVID 19, is an independent predictor of mortality $[9,10]$. Severe lymphopenia, with absolute count of less than $0.6 \times 10^{9} / \mathrm{L}$, was also found to be an important indicator for intensive care support in patients [11]. Lymphopenia in critically ill children is reported to be less common in comparison to adults with COVID-19 [12]. However, clinicians should keep in mind that higher neutrophil count has also been shown to be associated with increased risk of ARDS and death [10].

Hepatosplenomegaly, seen in other HLH syndromes due to direct infiltration of macrophages and lymphocytes [3], has not yet been reported in COVID-19 patients. Likewise, there are no reports of hemophagocytosis in bone marrow aspirate in these patients. Notably, hemophagocytosis is not a specific finding for sHLH and it can be seen in viral infections as well [13]. Hypertriglyceridemia, believed to be due to inhibition of lipoprotein lipase by TNF- $\alpha$, has not been documented in studies on adults, but it has been found in children [4]. Viral infections per se can also lead to hyperferritinemia suggesting a very low positive predictive value for diagnosing HLH with ferritin values [14]. Extremely high ferritin level $(>10,000 \mathrm{ng} / \mathrm{mL})$, which can detect sHLH/cytokine storm with around $95 \%$ sensitivity and specificity [3], has not been reported in adults or children $[1,2,8]$.

Low fibrinogen level, with its high specificity for HLH diagnosis, helps to distinguish HLH subset from critically ill patients with sepsis. Interestingly, a recent study in COVID-19 pneumonia patients showed that Ddimer was significantly higher in non-survivors on admission than in survivors, but fibrinogen was not significantly different between two groups [15]. Fibrinogen was found to decline very late during hospitalization in non-survivors $[10,15]$. There is increased production of fibrinogen, an acute phase reactant, in hyper-inflammatory condition. Fibrinogen level can remain normal despite increased consumption by circulating microthrombins in early hypercoagulable state of severe COVID-19. Hence, rather than the finding of low fibrinogen level, rising D-dimer value is more likely to detect the hyper-inflammatory state in COVID19 early in its course. Nonetheless, regular monitoring of fibrinogen would help guide clinicians to initiate cryoprecipitate infusion in case of bleeding with a drop in fibrinogen below $1.5 \mathrm{~g} / \mathrm{L}$.

A prospective study to diagnose sHLH subset in COVID-19 patients is underway using modified HLH2004 guidelines (NCT04347460). HScore, being a more sensitive tool, has advantages over HLH-2004 criteria for early detection of COVID-19 hyperinflammatory state. In absence of bone marrow examination in critical care setting, it is also pragmatic to consider a lower HScore threshold (see table 1) in case of strong clinical suspicion to avoid delay in interventions. In fact, use of intravenous immunoglobulin in children with severe COVID-19 looks promising [2]. At the same time, over diagnosing $\mathrm{HLH}$ and treating them with steroids may be counterproductive. Thus, HScore should only be used as a complement to clinical judgment on immunosuppression in severe COVID-19.

\section{Immunosuppression: A Word of Caution For} Glucocorticoids

Due to lack of efficacious antiviral therapies, timely administration of glucocorticoid therapy, as in other sHLH 
syndromes, indeed holds the promise to prevent development or further progression of ARDS and multiorgan dysfunction in COVID-19 with impending cytokine storm. The World Health Organization (WHO) does not advocate adjunctive use of steroids in critically ill COVID19 patients till now, unless indicated for other reasons, such as adrenal insufficiency [16]. 'Surviving sepsis guideline' has suggested (weak recommendation) the use of short course of low dose glucocorticoids in mechanically ventilated moderate-to-severe ARDS in adults apart from its usual recommendation in refractory septic shock [17]. However, there is no recommendation regarding use in children. Glucocorticoids for long duration in high doses can lead to increased ventilator dependence, osteonecrosis and poor cognitive outcomes in children. Hence, in early ARDS with suggestion of cytokine storm, the protocol of using glucocorticoids in lower doses (less than methylprednisolone equivalent dose $1-2 \mathrm{mg} / \mathrm{kg} /$ day) and short duration (5 days) is acceptable [18]. RECOVERY is a randomized trial currently in recruitment phase that aims to investigate whether treatment with lopinavir-ritonavir, hydroxy-chloroquine, azithromycin, corticosteroids or tocilizumab prevents mortality in children and adults with severe COVID-19 (NCT04381936). Of note, corticosteroid in the form of oral (liquid or tablets) or intravenous low dose dexamethasone daily for maximum 10 days is being administered in this trial (prednisolone in case of pregnant or breastfeeding mothers). Interim analysis of this trial has found significant mortality benefits with low dose dexamethasone in patients requiring oxygen or ventilator support; although, complete data is still awaited.

Nonetheless, delayed viral clearance, worsening of preexisting diabetes and poor outcomes in severe ARDS remain the concerns for glucocorticoid use in COVID-19 illness with severe ARDS [19]. Glucocorticoid use may further interfere with the ability to replenish lymphocyte pool in patients with severe lymphopenia and compromise on chances of survival. Hence, individualization depending on lymphocyte count is warranted with regular monitoring for dyselectrolytemia, hyperglycemia and serial differential count. Clinicians also need to be vigilant about the possibility of unmasking of Critical illness-related corticosteroid insufficiency (CIRCI) following treatment withdrawal in these patients [20].

Intravenous immunoglobulin alone or in combination with glucocorticoids or plasma exchange may also be beneficial in children with this infection-associated HLH/ cytokine storm [3]. IVIG has been recommended as a part of trials with variable doses, including, $1.0 \mathrm{~g} / \mathrm{kg} / \mathrm{d}$ for 2 days, or $400 \mathrm{mg} / \mathrm{kg} / \mathrm{d}$ for 5 days, $0.2 \mathrm{~g} / \mathrm{kg} / \mathrm{d}$ for $3-5$ days, or $2 \mathrm{~g} / \mathrm{kg} / \mathrm{d}$ infusion for 1-3 days. Data is still insufficient to recommend its use in HLH/cytokine storm with ARDS. Although recent prospective series on critically ill children has shown good outcomes with IVIG, randomized controlled trials are needed to draw validated conclusions [2]. However, for children with hyperinflammatory vasoplegic shock like presentation in recovery phase resembling atypical Kawasaki disease, IVIG $2 \mathrm{~g} / \mathrm{kg}$ within 24 hours of admission is warranted with vasopressor support and intravenous antibiotics cover [4].

Since significantly higher IL-6 was found in nonsurvivors compared to COVID-19 survivors [9], tocilizumab (anti-IL6 receptor antibody) has been widely used in many countries; but clinical evidence is still insufficient to recommend its use [16,17]. Clinical outcomes of tocilizumab administration will also be evaluated in hospitalized cancer patients of all ages with severe COVID-19 disease (NCT04370834). Other immunosuppressive drugs, eculizumab (anti-C5), siltuximab (anti-IL6), sarilumab (anti-IL6 receptor), anakinra (IL1 receptor antagonist), ruxolitinib \& baricitinib (JAK1-2 inhibitors), adalimumab (anti-TNF), meplazumab (anti-CD147) and ixekizumab (anti-IL 17A) had also been included in various clinical trials, mostly for patients above 12 years of age [21].

\section{COVID-19-ASSOCIATED COAGULOPATHY}

Apart from the cytokine storm, another distinctive feature noted in severe COVID-19 cases was development of COVID-19-associated coagulopathy (CAC). Unlike acutely ill patients with decompensated form of disseminated intravascular coagulation (DIC) who have high risk of bleeding, this coagulopathy resembles hypercoagulable state of compensated chronic DIC [22]. This can be attributed to the distinct thromboelastography findings of high fibrinogen and high factor VIII activity in these patients [23]. Tang et al. [12] reported that $71.4 \%$ of non-survivors had overt DIC based on ISTH DIC diagnostic criteria (Table I) in contrast to $0.6 \%$ of the survivors. Notably, in one recent large case series, none of the patients with thromboembolic events developed overt DIC [24]. Hence, it is important to screen these patients for a new category, namely sepsis induced coagulopathy (SIC), which is believed to precede overt DIC; therapeutic anticoagulant use is more likely to yield benefits in this early phase [25]. In support of this rationale, in a cohort of 183 patients with age range 14-94 years, prophylactic heparin use for $\geq 7$ days has been shown to be associated with significant reduction in 28-day mortality in patients with SIC score $\geq 4(40.0 \%$ vs. $64.2 \%)$ or D-dimer $>3 \mu \mathrm{g} /$ $\mathrm{mL}$, that is, 6-fold of upper limit (32.8\% vs. 52.4\%) [26]. 
Table I Scoring Systems for COVID-19-associated HLH and Coagulopathy

\begin{tabular}{|c|c|c|}
\hline HScore* [5] & $\begin{array}{l}\text { ISTH DIC } \\
\text { score }^{\#}[25]\end{array}$ & SIC scoret [25] \\
\hline $\begin{array}{l}\text { Temperature } \\
<38 \cdot 4^{\circ} \mathrm{C}: 0 \\
38 \cdot 4^{\circ} \mathrm{C}-39 \cdot 4^{\circ} \mathrm{C}: 33 \\
>39 \cdot 4^{\circ} \mathrm{C}: 49\end{array}$ & - & $\begin{array}{r}\text { Total SOFA score } \$ \\
1: 1 \\
\geq 2: 2\end{array}$ \\
\hline $\begin{array}{l}\text { Organomegaly } \\
\text { None: } 0 \\
\text { Hepato/splenomegaly: } \\
\text { Both: } 38\end{array}$ & $\begin{array}{l}- \\
23\end{array}$ & - \\
\hline $\begin{array}{l}\text { Cytopenias }{ }^{\wedge} \\
1 \text { lineage: } 0 \\
2 \text { lineages: } 24 \\
3 \text { lineages: } 34\end{array}$ & $\begin{array}{l}\text { Platelet count } \\
>100 \times 10^{9} / \mathrm{L}: 0 \\
50-100 \times 10^{9} / \mathrm{L}: 1 \\
<50 \times 10^{9} / \mathrm{L}: 2\end{array}$ & $\begin{array}{r}\text { Platelet count } \\
>150 \times 10^{9} / \mathrm{L}: 0 \\
100-150 \times 10^{9} / \mathrm{L}: 1 \\
<100 \times 10^{9} / \mathrm{L}: 2\end{array}$ \\
\hline $\begin{array}{l}\text { Triglycerides, } \mathrm{mmol} / \mathrm{L} \\
<1.5: 0 \\
1.5-4: 44 \\
>4 \cdot 0: 64\end{array}$ & $\begin{array}{l}\text { PT prolongation } \\
<3 \mathrm{~s}: 0 \\
3-6 \mathrm{~s}: 1 \\
>6 \mathrm{~s}: 2\end{array}$ & $\begin{array}{r}I N R \\
1.2-1.4: 1 \\
>1.4: 2\end{array}$ \\
\hline $\begin{array}{l}\text { Fibrinogen, } g / L^{* *} \\
>2 \cdot 5: 0 \\
\leq 2 \cdot 5: 30\end{array}$ & $\begin{array}{l}\text { Fibrinogen } \\
>1.0 \mathrm{~g} / \mathrm{L}: 0 \\
\leq 1.0 \mathrm{~g} / \mathrm{L}: 1\end{array}$ & - \\
\hline $\begin{array}{l}\text { Ferritin, } n g / m L \\
<2000: 0 \\
\text { 2000-6000: } 35 \\
>6000: 50\end{array}$ & $\begin{array}{l}D \text {-dimer } \\
\text { No increase: } 0 \\
\text { Moderate increase: } 2 \\
\text { Strong increase: } 3\end{array}$ & 2 \\
\hline $\begin{array}{l}S G O T, \mathrm{IU} / \mathrm{L} \\
<30: 0 \\
\geq 30: 19\end{array}$ & - & - \\
\hline $\begin{array}{l}\text { Bone marrow aspiratio } \\
\text { Hemophagocytosis: } 35 \\
\text { No: } 0\end{array}$ & $n^{\# \#}$ & - \\
\hline $\begin{array}{l}\text { Immunosuppression } t \bar{t} \\
\text { Yes: } 18 \\
\text { No: } 0\end{array}$ & - & - \\
\hline
\end{tabular}

*HScore $>169$ is $93 \%$ sensitive and $86 \%$ specific for HLH in adults [5]. To obtain similar specificity, HScore cut-off $>131$ had $94 \%$ sensitivity, whereas HScore $>120$ was $100 \%$ sensitive and $80 \%$ specific to detect HLH in children at initial presentation [6]. 'Cytopenias - (hemoglobin $\leq 9 \cdot 2 \mathrm{~g} / \mathrm{dL}$, white blood cell $\leq 5000 / \mathrm{mm}^{3}$ or platelet $\left.\leq 110,000 / \mathrm{mm}^{3}\right)$; ${ }^{* *}$ More emphasis on high $D$-dimer $(>3 \mu \mathrm{g} / \mathrm{mL})$ may be given instead for COVID-19 associated hyper-inflammatory state; \#\# Ine absence of BMA in critical care setting, it is prudent to consider a lower HScore threshold based on clinical judgement to avoid delay in immunosuppression; ${ }^{\dagger} H I V$ positive or on long term immunosuppressive therapies (i.e. glucocorticoids, cyclosporine, azathioprine); " ISTH DIC score $\geq 5$ is $88 \%$ sensitive and $96 \%$ specific for overt DIC; ; SIC score $\geq 4$ is diagnostic for SIC; ${ }^{\$}$ Total SOFA (pediatric sequential organ failure assessment) score is the sum of 4 items (respiratory SOFA, cardiovascular SOFA, hepatic SOFA, renal SOFA). Validated pediatric SOFA (pSOFA) score with age-adjusted variables should be used in children [39]; ISTH: International Society on Thrombosis and Haemostasis; DIC: disseminated intravascular coagulation; SIC: Sepsis induced coagulopathy; PT: Prothrombin time; INR: International normalized ratio; SGOT: Serum glutamic oxaloacetic transaminase.
International Society on Haemostasis and Thrombosis (ISTH) interim guidance recommends measuring Ddimers, prothrombin time (PT) and platelet count in all patients for risk stratification and subsequent management plan [27].

\section{Anticoagulant Use: Where Do We Stand?}

ISTH interim guidance has recommended only prophylactic low molecular weight heparin (LMWH) to all hospitalized patients with COVID-19 in absence of active bleed or thrombocytopenia (platelet count $<25 \times 10^{9} / \mathrm{L}$ ) [27]. Anti-inflammatory properties of heparin are believed to offer an additional benefit in this pro-inflammatory milieu. Based on existing literature regarding severe hypercoagulable state of non-survivors with COVID-19, Barrett, et al. [28] have strongly advised to consider therapeutic anticoagulation with unfractionated heparin to prevent life threatening micro and macro-vascular thrombosis [28]. It is to be noted that one child with SARS CoV-2 infection did succumb to extensive ischemic cerebro-vascular stroke in a recent case series [4]. Chinese expert groups had issued guidance regarding therapeutic unfractionated heparin use (UFH at a rate of 3-15 IU/kg/h) based on high fibrin degradation product (FDP $\geq 10 \mathrm{~g} / \mathrm{mL}$ ) or D-dimer concentration $(\geq 5 \mu \mathrm{g} / \mathrm{mL})$, even in absence of documented thromboembolism [29]. As an alternative, LMWH in higher than normal prophylactic doses is also being used as thrombo-prophylaxis in adults with critical COVID-19 illness in many hospitals around the world.

Current evidence must be extrapolated with caution in the pediatric population. Data on LMWH use in population below 14 years of age is lacking in the large study that guided the interim recommendations [26]. Based on preliminary data from children who recovered from critical illness, none of them received prophylactic heparin during hospitalization [2]. In the case series presenting with Kawasaki shock syndrome-like picture with cytokine storm and high D-dimer, six children were rather advised high dose anti-platelet (aspirin $50 \mathrm{mg} / \mathrm{kg}$ ); only one patient received heparin [4]. Heparin is generally avoided in children with hypercoagulable phase of DIC due to its potential adverse effect of bleeding, except for incident 'symptomatic' thrombi or acral ischemia [30]. Of note, skin manifestations resembling chilblains involving acral parts ('COVID toes') have appeared to be frequent among children and young population in recent literature [31]. This could be due to a direct virus-mediated endothelial damage, vasculitis or micro-thrombosis. Skin lesions in four children recovered without any sequelae in one case series [31]. Although elevated D-dimer along with the 
clinical features in one child did suggest vaso-occlusion owing to micro-thrombus, it was not symptomatic enough to warrant anticoagulant use [31].

In absence of data on prophylactic anticoagulant use in children, timely immunosuppression remains the key to halt the immune-thrombosis model of multi-organ dysfunction. In persistent hyperinflammatory state with high fibrinogen and increasing D-dimer trend, a course of IVIG or tocilizumab should be strongly considered as per standard pediatric intensive care protocol [32]. A recent case-report from India [33] reported the successful use of tocilizumab ( $8 \mathrm{mg} / \mathrm{kg}$ intravenous over $2 \mathrm{~h}$ ) as second line agent following single dose of IVIG $(2 \mathrm{~g} / \mathrm{kg})$ in an 8 -yearold child with COVID-19-associated hyperinflammatory syndrome. Plasma exchange may also be considered to salvage the situation in this setting.

Even if the clinical decision is made to administer anticoagulation in suspicion of thrombosis in persistent hyperinflammatory state and DIC, continuous intravenous infusion of UFH should be started with a low dose (5-10 U/kg/hour) and up-titrated slowly if required [30]. In addition to its advantage due to ease of titration, the anticoagulant effect also wears off quickly with stoppage. Activated partial thromboplastin time (aPTT) should be regularly monitored. UFH should be stopped in case of bleeding or high aPTT (more than 1.5 times upper normal level) [29]. For children with DIC, loading doses of heparin are generally avoided [30]. There is controversy regarding the type of heparin to be used in adults, since high fibrinogen and anti-thrombin deficiency in COVID-19 may lead to resistance to UFH [34]. Although LMWH has been used in adults with DIC, there is limited data to assess its efficacy in children with DIC [30]. Nonetheless, in case of symptomatic 'documented' thrombotic event or acro-ischemia, UFH is the ideal agent to use in pediatric intensive care setting, especially for those with renal insufficiency. For reasons unknown, heparin-induced thrombocytopenia seems to occur rarely in children [35].

One large-scale study on recombinant activated protein $\mathrm{C}$ (drotrecogin alpha) in patients with sepsis and DIC led to its abandonment from clinical use due to multiple reasons, notably, timing, dosing, efficacy and significant bleeding as a side effect [36]. However, studies are ongoing to evaluate other potential molecules in patients with sepsis induced coagulopathy or DIC. The preliminary success of recombinant soluble thrombomodulin in DIC and clinical states associated with endothelial dysfunction has shown promise with its tolerable side effect profile [37]. SARS CoV-2 enters the pulmonary epithelium by binding to ACE2; ACE2 in turn is cleaved and activated by host transmembrane serine protease 2 (TMPRSS2). Nafamostat, a TMPRSS2inhibitor that also potently inhibits thrombin and plasmin, has been identified as a potential therapy in patients with COVID-19 and DIC [38].

Even though bleeding is rare in COVID-19 associated coagulopathy, stringent monitoring of surgical sites and orifices is needed for patients on invasive ventilation or extracorporeal membrane oxygenation (ECMO) or continuous renal replacement therapy (CRRT). If bleeding ensues, similar transfusion principles for DIC/SIC as per ISTH guidelines should be followed to keep platelet count above $50 \times 10^{9} / \mathrm{L}$, fibrinogen above $2 \mathrm{~g} / \mathrm{L}$ and $\mathrm{PT}$ ratio below 1.5 [27].

\section{CONCLUSIONS}

COVID-19-associated coagulopathy resembles hypercoagulable state of DIC with rare reports of bleeding. All moderate-to-severe COVID-19 patients, especially with documented thrombocytopenia (platelet count $<150 \times 10^{9} / \mathrm{L}$ ) or acral manifestations, should undergo regular screening with SIC score, followed by screening for overt DIC. Unfractionated heparin infusion should be used in children with symptomatic thrombotic event under intensive monitoring. Although the presence of distinct COVID-19-HLH syndrome remains debatable, it is evident that a systemic hyper-inflammatory state has a significant role to play in 'immunothrombosis' model of multi-organ dysfunction and Kawasaki disease shock syndrome-like presentation in children. Interim analysis of RECOVERY trial has recently found mortality benefit with low dose dexamethasone for patients requiring oxygen or ventilator support. Immunosuppression with intravenous immunoglobulin has also shown favorable outcomes in critically ill children in preliminary studies. However, patient selection for immunosuppression should be done judiciously due to absence of wellcharacterized criteria for early identification of COVID19-related cytokine storm. In view of the limitations of HScore in this context, it may be prudent to consider using 'high D-dimer' ( $>3 \mu \mathrm{g} / \mathrm{mL}$ ) instead of 'low fibrinogen' to facilitate early diagnosis. HScore should only used as a complement to clinical judgment for instituting immunosuppressive therapies in severe COVID-19.

Funding: None; Competing interest: None stated.

\section{REFERENCES}

1. Huang C, Wang Y, Li X, Ren L, Zhao J, Hu Y, et al. Clinical features of patients infected with 2019 novel coronavirus in Wuhan, China. Lancet. 2020;395:P497P506. 
2. Sun D, Li H, Lu XX, Xiao H, Ren J, Zhang FR, Liu ZS. Clinical features of severe pediatric patients with coronavirus disease 2019 in Wuhan: a single center's observational study. World J Pediatri. 2020;19:1-9.

3. George MR. Hemophagocytic lymphohistiocytosis: Review of etiologies and management. J Blood Med. 2014;5:69-86.

4. Riphagen S, Gomez X, Gonzalez-Martinez C, Wilkinson $\mathrm{N}$, Theocharis P. Hyperinflammatory shock in children during COVID-19 pandemic. Lancet. 2020;S0140673631094-1.

5. Fardet L, Galicier L, Lambotte O, Marzac C, Aumont C, Chahwan $\mathrm{D}$, et al. Development and validation of the HScore, a score for the diagnosis of reactive hemophagocytic syndrome. Arthritis Rheumatol. 2014;66:2613-20.

6. Debaugnies F, Mahadeb B, Ferster A, Meuleman N, Rozen $\mathrm{L}$, Demulder A, et al. Performances of the H-Score for diagnosis of hemophagocytic lymphohistiocytosis in adult and pediatric patients. Am J Clin Pathol. 2016;145:862-70.

7. Mehta P, McAuley DF, Brown M, Sanchez E, Tattersall RS, Manson JJ. COVID-19: Consider cytokine storm syndromes and immunosuppression. Lancet. 2020;395:1033-4.

8. Chen G, Wu D, Guo W, Cao Y, Huang D, Wang H, et al. Clinical and immunological features of severe and moderate coronavirus disease 2019. J Clin Invest. 2020;13:2620-29.

9. Ruan Q, Yang K, Wang W, Jiang L, Song J. Clinical predictors of mortality due to COVID-19 based on an analysis of data of 150 patients from Wuhan, China. Intensive Care Med. 2020;1-4.

10. Terpos E, Ntanasis Stathopoulos I, Elalamy I, Kastritis E, Sergentanis TN, Politou M, et al. Hematological findings and complications of COVID 19. Am J Hematol. 2020.

11. Fan BE, Chong VC, Chan SS, Lim GH, Lim KG, Tan GB, et al. Hematologic parameters in patients with COVID-19 infection. Am J Hematol. 2020.

12. Ong JS, Tosoni A, Kim Y, Kissoon N, Murthy S. Coronavirus disease 2019 in critically Ill children: A narrative review of the literature. Pediatr Crit Care Med. 2020;21:662-6.

13. Zoller EE, Lykens JE, Terrell CE, Aliberti J, Filipovich $\mathrm{AH}$, Henson PM, et al. Hemophagocytosis causes a consumptive anemia of inflammation. J Exp Med. 2011; 208:1203-14.

14. Sackett K, Cunderlik M, Sahni N, Killeen AA, Olson AP. Extreme hyperferritinemia: Causes and impact on diagnostic reasoning. Am J Clin Pathol. 2016;145.646-50.

15. Tang N, Li D, Wang X, Sun Z. Abnormal coagulation parameters are associated with poor prognosis in patients with novel coronavirus pneumonia. J Thromb Haemost. 2020;18:844.

16. WHO. Clinical management of Severe Acute Respiratory Infection When Novel Coronavirus (nCoV) Infection is Suspected: Interim Guidance. Available from: https:// www.who.int/publications-detail/clinical-management-ofsevere-acute-respiratory-infection-when-novel-corona virus-(ncov)-infection-is-suspected. Accessed May 10, 2020 .

INDIAN PEDIATRICS
17. Alhazzani W, Møller MH, Arabi YM, Loeb M, Gong MN, Fan E, et al. Surviving sepsis campaign: guidelines on the management of critically ill adults with coronavirus disease 2019 (COVID-19). Intensive Care Med. 2020;46:854-87.

18. Qin YY, Zhou YH, Lu YQ, Sun F, Yang S, Harypursat V, et al. Effectiveness of glucocorticoid therapy in patients with severe novel coronavirus pneumonia: Protocol of a randomized controlled trial. Chin Med J (Engl). 2020;139:1080-6.

19. Russell CD, Millar JE, Baillie JK. Clinical evidence does not support corticosteroid treatment for 2019-nCoV lung injury. Lancet. 2020; 395:473-75.

20. Pal R, Banerjee M. COVID-19 and the endocrine system: Exploring the unexplored. J Endocrinol Invest. 2020:1-5.

21. Lythgoe MP, Middleton P. Ongoing clinical trials for the management of the COVID-19 pandemic. Trends Pharmacol Sci. 2020;S0165-6147:30070-5.

22. Connors JM, Levy JH. Thromboinflammation and the hypercoagulability of COVID-19. J Thromb Haemost. 2020;10.1111/jth.14849 [published online ahead of print].

23. Panigada $\mathrm{M}$, Bottino $\mathrm{N}$, Tagliabue $\mathrm{P}$, et al. Hypercoagulability of COVID-19 patients in intensive care unit. A report of thromboelastography findings and other parameters of hemostasis. J Thromb Haemost. 2020;10.1111/jth. 14850 [published online ahead of print].

24. Klok FA, Kruip MJHA, van der Meer NJM, et al. Incidence of thrombotic complications in critically ill ICU patients with COVID-19. Thromb Res. 2020;S0049-3848:30120-1.

25. Iba T, Levy JH, Warkentin TE, Thachil J, van der Poll T, Levi $\mathrm{M}$, et al. Diagnosis and management of sepsis induced coagulopathy and disseminated intravascular coagulation. J Thromb Haemost. 2019; 17:1989-94.

26. Tang N, Bai H, Chen X, Gong J, Li D, Sun Z. Anticoagulant treatment is associated with decreased mortality in severe coronavirus disease 2019 patients with coagulopathy. J Thromb Haemost. 2020;18:1094-1099

27. Thachil J, Tang N, Gando S, Falanga A, Cattaneo M, Levi $\mathrm{M}$, et al. ISTH Interim Guidance on Recognition and Management of Coagulopathy in COVID 19. J Thromb Haemost. 2020;18:1023-6.

28. Barrett CD, Moore HB, Yaffe MB, Moore EE. ISTH interim guidance on recognition and management of coagulopathy in COVID 19: A comment. J Thromb Haemost. 2020;10.1111/jth.14860 [published online].

29. Song JC, Wang G, Zhang W, Zhang Y, Li W-Q, Zhou Z, et al. Chinese expert consensus on diagnosis and treatment of coagulation dysfunction in COVID-19. MilMed Res. 2020;7:19.

30. Wong W, Glader B. Disseminated intravascular coagulation in infants and Children. Available from: https:/ /www.uptodate.com/ contents/ disseminatedintravascular-coagulation-in-infants-and-children. Accessed May 10, 2020.

31. Colonna C, Monzani NA, Rocchi A, Gianotti R, Boggio F, Gelmetti C. Chilblains like lesions in children following suspected Covid 19 infection. Pediatr Dermatol. 2020; 10.1111/pde. 14210 [published online ahead of print].

32. Ravikumar N, Nallasamy K, Bansal A, Angurana SK, Basavaraja GV, Sundaram M, et. al. Novel Coronavirus 
2019 (2019-nCoV) infection: Part I-Preparedness and management in the pediatric intensive care unit in resourcelimited settings. Indian Pediatr. 2020;57:324-34.

33. Balasubramanian S, Nagendran TM, Ramachandran B, Ramanan AV. Hyper-inflammatory syndrome in a child with covid-19 treated successfully with intravenous immunoglobulin and tocilizumab. Indian Pediatr. 2020;S097475591600180 [online ahead of print].

34. Thachil J, Tang N, Gando S, Falanga A, Levi M, Clark C, et al. Type and dose of heparin in Covid-19: Reply. J Thromb Haemost. 2020. [published online ahead of print].

35. Monagle P, Newall F. Management of thrombosis in children and neonates: Practical use of anticoagulants in children. Hematology Am Soc Hematol Educ Program. 2018;2018:399-404.
36. Ranieri VM, Thompson BT, Barie PS, Dhainaut JF, Douglas IS, Finfer S, et al. Drotrecogin alfa (activated) in adults with septic shock. N Engl J Med. 2012;366:2055-64.

37. Ito $\mathrm{T}$, Thachil J, Asakura $\mathrm{H}$, Levy JH, Iba $\mathrm{T}$. Thrombomodulin in disseminated intravascular coagulation and other critical conditions-A multi-faceted anticoagulant protein with therapeutic potential. Critical Care. 2019;23:280

38. Asakura H, Ogawa H. Potential of heparin and nafamostat combination therapy for COVID-19. J Thromb Haemost. 2020. [published online ahead of print].

39. Matics TJ, Sanchez-Pinto LN. Adaptation and validation of a pediatric sequential organ failure assessment score and evaluation of the sepsis-3 definitions in critically ill children. JAMA Pediatr. 2017;171:e172352. 